\title{
Ethoxysanguinarine inhibits viability and induces apoptosis of colorectal cancer cells by inhibiting CIP2A
}

\author{
LAN JIN $^{1 *}$, YUAN SI ${ }^{1 *}$, XING HONG ${ }^{1}$, PENGFEI LIU ${ }^{1}$, BEIBEI ZHU ${ }^{1}$, HUILIANG YU ${ }^{2}$, XINHUA ZHAO $^{1}$, \\ SHANSHAN QIN ${ }^{1}$, MENGYUAN XIONG ${ }^{1}$, YING LIU ${ }^{1}$, ZHIGUO LUO $^{3}$ and YANG GUO ${ }^{1}$ \\ ${ }^{1}$ Laboratory of Molecular Target Therapy of Cancer, Institute of Basic Medical Sciences, Hubei University of Medicine, \\ Shiyan, Hubei 442000; ${ }^{2}$ Hubei Province Key Laboratory of Conservation Biology for Shennongjia Golden Monkey, \\ Administration of Shennongjia National Park, Shennongjia Forestry Region, Hubei 442421; ${ }^{3}$ Department of \\ Clinical Oncology, Taihe Hospital, Hubei University of Medicine, Shiyan, Hubei 442000, P.R. China
}

Received October 9, 2017; Accepted February 13, 2018

DOI: $10.3892 /$ ijo.2018.4323

\begin{abstract}
Cancerous inhibitor of protein phosphatase $2 \mathrm{~A}$ (CIP2A) an endogenous inhibitor of protein phosphatase $2 \mathrm{~A}$ (PP2A), which can promote proliferation and transformation of several cancer types, has been shown to be a target for tumor therapy. The present study investigated the effects and underlying mechanisms of action of a novel natural compound, ethoxysanguinarine (Eth), on colorectal cancer (CRC) cells. MTT assay and flow cytometric assay found that Eth inhibited the viability and induced the apoptosis of the CRC cells. The inhibition of viability and activation of apoptosis was mediated through the Eth-induced decrease in CIP2A expression. Knockdown of CIP2A by RNA interference sensitized, whereas overexpression of CIP2A antagonized, Eth-induced viability inhibition and apoptosis. Furthermore, western blot analysis suggested that Eth inhibited phosphorylation of CIP2A downstream molecule protein kinase B via the activation of PP2A. CRC xenograft tests also confirmed the antitumor effect of Eth in vivo. These results advance our understanding of Eth-induced viability inhibition and apoptosis, implying the requirement for further investigation of Eth as a CIP2A inhibitor for cancer therapies.
\end{abstract}

Correspondence to: Dr Yang Guo, Laboratory of Molecular Target Therapy of Cancer, Institute of Basic Medical Sciences, Hubei University of Medicine, 30 Renmin Road, Shiyan, Hubei 442000, P.R. China

E-mail: guoyang_hbmu@foxmail.com

Dr Zhiguo Luo, Department of Clinical Oncology, Taihe Hospital, Hubei University of Medicine, 32 Renmin Road, Shiyan, Hubei 442000, P.R. China

E-mail: camelwhite@163.com

*Contributed equally

Key words: ethoxysanguinarine, colorectal cancer, cancerous inhibitor of protein phosphatase $2 \mathrm{~A}$, apoptosis, Akt

\section{Introduction}

Colorectal cancer (CRC) is the second most commonly diagnosed cancer in females and the third in males, with an estimated 1.4 million new cases and 693,900 associated mortalities occurring globally in 2012 (1). In China, CRC is the fifth leading cause of cancer-associated mortality (2). In developing countries, it is predicted that between the years 2015 and 2030, the incidence of CRC will increase by as much as $60 \%$ (3). Patients diagnosed at early stages have a better prognosis, with a survival rate of 5 years. However, numerous patients are often diagnosed at an advanced stage, therefore have low response and high recurrence rates (4). Chemotherapy is a common therapeutic option for advanced CRC, but has limited effectiveness, with a poor prognosis and cancer relapse (5). Therefore, there is a requirement to identify novel prognostic biomarkers and key target molecules for CRC treatment.

Cancerous inhibitor of protein phosphatase 2A (CIP2A), originally known as KIAA1524 or P90, has been cloned from patients with hepatocarcinoma (6). CIP2A functions as an oncoprotein that promotes the growth, transformation, drug resistance and maintenance of a malignant cellular phenotype of various cancer types, including CRC, head and neck cancer, oral squamous cell carcinoma, esophageal squamous cell carcinoma, breast cancer, gastric cancer, tongue cancer, prostate cancer lung cancer, cervical cancer and leukemia (6-10). CIP2A is a cellular inhibitor of protein phosphatase 2A (PP2A) (6). PP2A serves as a key tumor suppressor that regulates signaling pathways and has a high relevance in human cancer (11). As a tumor suppressor, PP2A serves a critical role in the regulation of survival, differentiation and cell-cycle progression by negatively regulating the phosphoinositide 3-kinase/protein kinase B (Akt) pathway and inactivating extracellular signal-regulated kinase/dual specificity mitogen-activated protein kinase kinase 1 family kinases (10). Therefore, CIP2A is considered as a prognostic indicator, and targeting CIP2A/PP2A/Akt may be attractive for cancer therapy.

Ethoxysanguinarine (Eth) is a benzophenanthridine alkaloid natural product that is mainly found in Macleaya cordata 
(Willd) R. Br. (also known as Bo Luo Hui) of the Papaveraceae family (12). In China, $M$. cordata is used as an herbal medicine and has been widely applied for a long time due to its wide bioactivities, e.g., antimicrobial, antifungal, antiinflammatory, antioxidant and antitumor activities (10,13-15). M. cordata has also been used for the treatment of insect stings or bites and tinea infection in China, Europe and North America (10). Eth is converted by sanguinarine upon crystallization with ammoniated ethanol during the isolation process (16). Eth is reported to exhibit antiviral activity by downregulation of the porcine reproductive and respiratory syndrome virus-induced cytopathic effect. However, the effect of Eth on human tumor cells remains unclear. To the best of our knowledge, only one study has investigated the effect of Eth on human tumor cells. In 2013, Liu et al (12) revealed that Eth can induce inhibitory effects and downregulate CIP2A in human lung cancer cells. The effect and mechanism of Eth in other cancer types requires investigation. The present study aimed to investigate the antitumor activities and the possible mechanisms of Eth against CRC.

\section{Materials and methods}

Patients. In total, 6 pairs of tumor and adjacent normal colon tissues were collected from patients (mean age, 63 years; age range, 48-76 years; sex ratio, 3:3) who were treated between July 2016 and July 2017 at the Department of Clinical Oncology, Taihe Hospital, Hubei University of Medicine (Shiyan, Hubei, China) and stored in liquid nitrogen until further use. Samples were ground in liquid nitrogen and suspended in lysis buffer [50 mM Tris- $\mathrm{HCl}(\mathrm{pH} 7.4), 150 \mathrm{mM}$ $\mathrm{NaCl}, 1 \%$ Triton $\mathrm{X}-100,1 \%$ sodium deoxycholate, $0.1 \%$ SDS, $1 \mathrm{mM}$ PMSF and complete protease inhibitor cocktail) and cleared by centrifugation $\left(14,000 \mathrm{x} \mathrm{g}\right.$ at $4^{\circ} \mathrm{C}$ for $\left.15 \mathrm{~min}\right)$. Proteins extracted from the tissues were used for western blotting. The tissue specimens were collected and used with the approval of the Institutional Review Board of Hubei University of Medicine (Shiyan, Hubei, China) and Taihe Hospital affiliated to Hubei University of Medicine (Shiyan, Hubei, China). Written informed consent was obtained from all patients.

Quantitative polymerase chain reaction ( $q P C R)$. The expression level of the $C I P 2 A$ gene was examined by qPCR. GAPDH was used as an endogenous control for each sample. Total RNA from SW620 or HT29 cells or patients' tissues were extracted using TRIzol reagent (Invitrogen; Thermo Fisher Scientifc, Inc., Waltham, MA, USA) according to the manufacturer's protocols. Total RNA ( $2 \mu \mathrm{g})$ and the ReverTra Ace qPCR RT kit (Toyobo Life Science, Osaka, Japan) was used for qPCR analysis of CIP2A. Reverse transcription occurred at $37^{\circ} \mathrm{C}$ for $15 \mathrm{~min}$ and $98^{\circ} \mathrm{C}$ for $5 \mathrm{~min}$, with storage at $-20^{\circ} \mathrm{C}$. RNA $(2 \mu \mathrm{g}), 4 \mu \mathrm{l}$ 5X RT Buffer, $1 \mu \mathrm{l}$ RT Enzyme mix, $1 \mu \mathrm{l}$ Primer mix and Nuclease-free Water were mixed to a $20-\mu 1$ total volume. The primers used in this study were as follows: CIP2A forward, 5'-TGCGGCACTTGGAGGTAATTTC-3' and reverse, 5'-AGCTCTACAAGGCAACTCAAGC-3'; and GAPDH forward, 5'-TGTTGCCATCAATGACCCCTT-3' and reverse, 5'-CTCCACGACGTACTCAGCG-3'. qPCR was performed using an ABI StepOnePlus ${ }^{\mathrm{TM}}$ Real-Time PCR system (Applied Biosystems; Thermo Fisher Scientific, Inc.) with the Power SYBR ${ }^{\circledR}$-Green PCR Master mix (Toyobo Life Science).
SYBR-Green PCR Master Mix (10 $\mu \mathrm{l})$, forward and reverse primers $(200 \mathrm{nM})$, cDNA template $(100 \mathrm{ng})$ and $\mathrm{ddH}_{2} \mathrm{O}$ were mixed to a $20-\mu 1$ total volume. PCR conditions consisted of the following: $95^{\circ} \mathrm{C}$ for $3 \mathrm{~min}, 95^{\circ} \mathrm{C}$ for $15 \mathrm{sec}$ and $60^{\circ} \mathrm{C}$ for $1 \mathrm{~min}$, for 40 cycles. The threshold cycle for each sample was selected from the linear range and converted to a starting quantity by interpolation from a standard curve generated on the same plate for each set of primers. The CIP $2 A$ mRNA levels were evaluated using the $2^{-\Delta \Delta \mathrm{Cq}}$ method, standardized to levels of GAPDH amplification (17). Each test was performed in triplicate.

Western blot analysis. SW620 or HT29 cells were harvested and lysed with RIPA buffer containing $50 \mathrm{mM}$ Tris, $150 \mathrm{mM}$ $\mathrm{NaCl}, 0.1 \%$ SDS, $0.5 \%$ deoxycholate, $1 \%$ NP-40, $1 \mathrm{mM}$ DTT, $1 \mathrm{mM} \mathrm{NaF,} 1 \mathrm{mM}$ sodium vanadate, $1 \mathrm{mM}$ PMSF (Sigma-Aldrich; Merck KGaA, Darmstadt, Germany) and a $1 \%$ protease inhibitor cocktail (Merck KGaA). Lysates from cells or the patients' tissues were normalized for total protein $(25 \mu \mathrm{g})$ and loaded on 8-12\% SDS-PAGE gels. Subsequently, the gels were electrophoretically transferred to polyvinylidene difluoride membranes (EMD Millipore, Billerica, MA, USA). Subsequent to being blocked with $5 \%$ skimmed milk at room temperature for $1 \mathrm{~h}$, the membranes were incubated at $4^{\circ} \mathrm{C}$ with primary antibodies overnight and washed three times with Tris-buffered saline containing 0.5\% Tween-20. The primary antibodies used were anti-CIP2A (1:500 dilution; cat. no. sc-80662), anti-phospho-Akt (S473) (1:500 dilution; catalog no. sc-7985), anti-Akt (1:500 dilution; cat. no. sc-8312) (all from Santa Cruz Biotechnology, Inc., Dallas, TX, USA), anti-caspase-3 (1:1,000 dilution; cat. no. 9662), anti- poly ADP ribose polymerase (PARP; 1:1,000 dilution; cat. no. 9542), anti-PP2A (1:1,000 dilution; cat. no. 2038) (all from Cell Signaling Technology, Inc., Danvers, MA, USA) and anti-GAPDH (1:5,000 dilution; cat. no. M20006; Abmart Co., Ltd., Shanghai, China). Subsequently the membranes were incubated with horseradish peroxidase-conjugated secondary antibody [1:10,000 dilution; catalog nos. E030120-01 (rabbit) and E030110-01 (mouse); EarthOx, LLC, San Francisco, CA, USA] at room temperature for $1.5 \mathrm{~h}$. Detection was performed using a SuperSignal ${ }^{\circledR}$ West Pico Trial kit (cat. no. QA210131; Pierce; Thermo Fisher Scientific, Inc.) (18). The defined sections of the film were scanned for image capture and quantification using Adobe Photoshop CS4 software (Adobe Systems Inc., San Jose, CA, USA) and ImageJ software (National Institutes of Health, Bethesda, MD, USA).

Reagents. Eth with a purity of up to $98 \%$, obtained from Shanghai Yuanye Bio-Technology Co., Ltd. (Shanghai, China), was dissolved in dimethyl sulfoxide (Sigma-Aldrich; Thermo Fisher Scientific, Inc.) at a stock solution of $50 \mathrm{mM}$ and stored at $-20^{\circ} \mathrm{C}$.

Cell culture. A total of 4 CRC cell lines, SW620, SW480, HT29 and HCT116, were procured from the American Type Culture Collection (Manassas, VA, USA). SW620 and SW480 cells were grown in Leibovitz's-15 (L-15) medium (Gibco; Thermo Fisher Scientific, Inc.) containing 10\% fetal bovine serum (FBS; HyClone; GE Healthcare Life Sciences, Logan, UT, USA) and antibiotics. All cells were then incubated in a humidified atmosphere without $\mathrm{CO}_{2}$ at $37^{\circ} \mathrm{C}$. HT29 and HCT116 cells were grown in Dulbecco's modified Eagle's 
medium (DMEM; Gibco; Thermo Fisher Scientific, Inc.) containing with $10 \%$ FBS and antibiotics, and incubated at $37^{\circ} \mathrm{C}$ in an incubator containing $5 \% \mathrm{CO}_{2}$.

Cytotoxicity assay and cell viability. An MTT assay (MTT dissolved in phosphate-buffered saline) was used to calculate cell cytotoxicity. A total of $5 \times 10^{5}$ cells were seeded into a 96-well plate and pre-cultured with L-15 or DMEM for $24 \mathrm{~h}$, then treated with Eth $(0.5-12 \mu \mathrm{M})$ for $24 \mathrm{~h}$. The absorbance was measured at $490 \mathrm{~nm}\left(\mathrm{~A}_{490}\right)$ with an enzyme immunoassay analyzer (BioTek Instruments, Inc., Winooski, VT, USA), and the inhibition rate was calculated as followed: Inhibition rate $(\%)=\left(\right.$ average $\mathrm{A}_{490}$ of the control group-average $\mathrm{A}_{490}$ of the experimental group)/(average $\mathrm{A}_{490}$ of the control group-average $\mathrm{A}_{490}$ of the blank group) $\mathrm{x} 100$. Cell viability was estimated by trypan blue dye exclusion as described previously $(19,20)$.

Soft-agar colony formation assay. This assay was performed in 6-well plates containing $0.6 \%$ agarose and $10 \%$ FBS. A total of $1 \times 10^{3}$ cells were seeded in $1 \mathrm{ml} \mathrm{L}-15$ or DMEM containing $10 \%$ FBS with $0.3 \%$ low-melting-point agarose (Amresco, LLC, Solon, OH, USA), and layered onto the base. After 2 weeks, the plates were stained with $0.2 \%$ gentian violet at room temperature for $20 \mathrm{~min}$ and the number of colonies was counted under a light microscope (IX70; Olympus Corporation, Tokyo, Japan) (18).

Apoptosis determination by DAPI staining. Approximately $5 \times 10^{5}$ cells/well in a 12-well plate were treated with Eth $(0-3 \mu \mathrm{M}$ for SW620 cells; 0-5 $\mu \mathrm{M}$ for HT29 cells) for $24 \mathrm{~h}$. The cells in each treatment and control were then stained with DAPI at room temperature for $8 \mathrm{~min}$. Representative images were captured using fluorescence microscopy, as previously described (21).

Cycloheximide (CHX) treatment. SW620 (or HT29) cells were treated with $50 \mu \mathrm{g} / \mathrm{ml} \mathrm{CHX}$ in the absence or presence of $2 \mu \mathrm{M}$ (or $4.5 \mu \mathrm{M}$ ) Eth for 0, 6, 8 and $12 \mathrm{~h}$, and cell lysates were harvested for western blot assays.

Flow cytometric assays for Annexin $V(A V)$. Cell apoptosis was evaluated using an AV-FITC kit (BD Biosciences, San Jose, CA, USA). According to the manufacturer's instructions, the samples were subjected to flow cytometry to assess the apoptotic cells, as described previously (22).

Transfection of small interfering RNA (siRNA). Two sequences of CIP2A siRNA were designed and then synthesized by Shanghai GenePharma Co., Ltd., (Shanghai, China), referred to as siRNA1 and siRNA2. The sequences of the CIP2A siRNA were as follows: CIP2A siRNA1, 5'-CUGUGGUUGU GUUUGCACUTT-3'; CIP2A siRNA2, 5'-ACCAUUGAUAUC CUUAGAATT-3'; and negative control siRNA, 5'-UUCUCCG AACGUGUCACGUTT-3'. CRC cells were transfected with $100 \mathrm{nM}$ siRNA using Lipofectamine ${ }^{\circledR} 2000$ (Invitrogen; Thermo Fisher Scientific, Inc.) according to the manufacturer's protocols. At $48 \mathrm{~h}$ post-transfection, the cells were used for western blotting and cell viability analysis.

Transfection of DNA. The pOTENT-1-CIP2A expression plasmid was purchased from Youbio Co. (Changcha, China).
The pOTENT-1-CIP2A plasmid ( $1 \mu \mathrm{g} / \mu \mathrm{l})$ was transfected into CRC cells using Lipofectamine ${ }^{\circledR} 3000$ transfection reagent (Invitrogen; Thermo Fisher Scientific, Inc.) following the manufacturer's protocols.

PP2Aactivityassay. PP2A phosphatase activity was tested using a PP2A immunoprecipitation phosphatase assay kit (Upstate Biotechnology, Inc., Lake Placid, NY, USA). According to the manufacturer's instructions, $100 \mu \mathrm{g}$ protein isolated from the cells and $4 \mu \mathrm{g}$ anti-PP2A monoclonal antibody (1:100 dilution; cat. no. 2038; Cell Signaling Technology, Inc.) were incubated together at $4^{\circ} \mathrm{C}$ overnight. Protein A agarose beads $(40 \mu \mathrm{l})$ were added to the mixture and incubated at $4^{\circ} \mathrm{C}$ for $2 \mathrm{~h}$, and then the beads were collected and washed three times with $700 \mu$ licecold TBS and once with $500 \mu 1 \mathrm{Ser} / \mathrm{Thr}$ Assay Buffer (Upstate Biotechnology, Inc.). The beads were further incubated with $750 \mathrm{mM}$ phosphopeptide in assay buffer at $30^{\circ} \mathrm{C}$ for $10 \mathrm{~min}$ with continuous agitation. Malachite Green Phosphate Detection Solution $(100 \mu \mathrm{l})$ was added and the absorbance at $650 \mathrm{~nm}$ was measured, as described previously (23).

Human CRC xenograft experiments. Equal numbers of female and male $(\mathrm{n}=20), 5$-week-old, nude immunodeficient mice (nu/nu) (weighing $\sim 16 \mathrm{~g}$ ) were purchased from Hunan SJA Laboratory Animal Co., Ltd. (Changsha, China), and maintained and monitored in a specific pathogen-free environment (temperature, $22-24^{\circ} \mathrm{C}$; barrier environment; $12 \mathrm{~h}$ dark $/ 12 \mathrm{~h}$ light cycle; sterile water and full nutritive feed). All animal studies were conducted according to protocols approved by the Hubei University of Medicine Animal Care and Use Committee, complying with the rules of Regulations for the Administration of Affairs Concerning Experimental Animals (approved by the State Council of China). The mice were injected subcutaneously with SW620 cells $\left(5 \times 10^{6}\right)$ suspended in $100 \mu 1$ L-15 medium into the right flank of each mouse. Treatments were started when the tumors reached a palpable size. Mice were randomly divided into two groups and treated with Eth $(0.5 \mathrm{mg} / \mathrm{kg} ; \mathrm{n}=8)$, or vehicle control $(\mathrm{n}=8)$ for 4 weeks. Caliper measurements of the longest perpendicular tumor diameters were performed twice a week to estimate the tumor volume, using the following formula: $4 \pi / 3 \times(\text { width } / 2)^{2} \times($ length/2), representing the 3 -dimensional volume of an ellipse. Animals were sacrificed when tumors reached $1.5 \mathrm{~cm}$ in diameter. At the time of death, tumors were excised for western blotting.

Statistical analysis. All statistical analyses were conducted using GraphPad Prism 5 (GraphPad Software, Inc.,La Jolla,CA, USA) and SPSS 22.0 software for Windows (IBM Corporation Armonk, NY, USA). Results from three independent experiments were presented as the mean \pm standard deviation unless otherwise noted. Statistically significant values were compared using Student's t-test of unpaired data or one-way analysis of variance and Bonferroni's post hoc test. $\mathrm{P}<0.05$ was used to indicate a statistically significant difference.

\section{Results}

High expression of CIP2A and high phosphorylation of Akt in human CRC tissues. qPCR and western blot analyses were used to detect the mRNA and protein levels of CIP2A and 
its downstream molecule Akt in the human CRC tissues of 6 patients. CIP2A exhibited higher expression in all tumor tissues, with the exception of case no. 2, compared with the patient-matched adjacent normal colonic tissues at the mRNA and protein levels (Fig. 1). Similarly, Akt phosphorylation was elevated in 6 cases (Fig. 1B).

Eth inhibits the viability of CRC cells. The effect of Eth (Fig. 2A) on cell viability was investigated with the four CRC cell lines, SW620, SW480, HT29 and HCT116. An MTT assay showed that Eth exhibited moderate cytotoxicity to these cell lines, with an half maximal inhibitory concentration of 3.57-9.37 $\mu \mathrm{M}$ (Fig. 2B; Table I). As shown in Fig. 2C and D, Eth was effective in inhibiting the growth of the SW620 and HT29 CRC cells. Trypan blue exclusion assay showed that Eth rapidly reduced the number of viable SW620 (Fig. 2E) and HT29 (Fig. 2F) cells in a dose- and time-dependent manner. Colony formation activity showed that Eth significantly inhibited the clonogenic ability of SW620 cells (Fig. 2G and H).

Eth induces apoptosis of CRC cells in a caspase-dependent manner. The study next tested whether Eth is able to induce apoptosis. DAPI staining showed that Eth induced chromatin condensation and fragmentation in SW620 and HT29 cells, which are typical apoptotic nuclear morphological changes (Fig. 3A and B). AV-FITC/propidium iodide staining and flow cytometry assays were then used to confirm that Eth induced apoptosis in the SW620 and HT29 cells (Fig. 3C and D). Furthermore, western blot analysis detected that Eth induced a decrease in the prosomal form of caspase-3 (pro-casp-3) and cleavage of PARP (cleaved-PARP) in a dose-dependent manner in the SW620 (Fig. 3E) and HT29 (Fig. 3F) cells. These results suggested that Eth induced caspase-dependent apoptosis in CRC cells.

Eth treatment inhibits the expression of CIP2A in CRC cells. The effect of Eth on the expression of CIP2A was investigated by western blotting. It was found that treatment with Eth at 1-3 $\mu \mathrm{M}$ could downregulate CIP2A expression in SW620 cells (4.5-5 $\mu \mathrm{M}$ in HT29 cells) (Fig. 4A and 4B). Fig. 4A shows that the protein level of CIP2A was decreased in SW620 cells treated with Eth at $1 \mu \mathrm{M}$ and then much less detectable in cells treated with Eth at $2 \mu \mathrm{M}$. In addition, treatment of HT29 cells with Eth at $4.5 \mu \mathrm{M}$ induced an apparent downregulation of CIP2A (Fig. 4B). Furthermore, it was found that Eth induced downregulation of CIP2A in a time-dependent manner (Fig. 4C and D). Next, qPCR was used to investigate whether Eth affected $C I P 2 A$ gene transcription. The result showed that Eth treatment significantly downregulated CIP2A mRNA expression (Fig. 4E and F). These results indicate that Eth downregulates $C I P 2 A$ transcription. Since Eth induced a relatively rapid degradation of CIP2A, we speculated that Eth may also influence CIP2A stability. Thus, a protein synthesis inhibitor, cycloheximide (CHX), was next used to block protein synthesis, and the results showed that CIP2A was stable under $\mathrm{CHX}$ treatment for at least $12 \mathrm{~h}$. However, when cells were co-incubated with $\mathrm{CHX}$ and Eth, CIP2A expression was downregulated in $6 \mathrm{~h}$ (Fig. 4G). Conclusively, these results indicate that Eth decreases $C I P 2 A$ transcription and induces CIP2A proteolysis.
Table I. $\mathrm{IC}_{50}$ values of Eth in colorectal cancer cell lines.

\begin{tabular}{ll}
\hline Cell line & $\mathrm{IC}_{50}, \mu \mathrm{M}$ \\
\hline SW480 & $9.37 \pm 0.83$ \\
HCT116 & $7.19 \pm 0.73$ \\
HT29 & $6.55 \pm 0.50$ \\
SW620 & $3.57 \pm 0.54$
\end{tabular}

The cells were treated with Eth at various concentrations for $24 \mathrm{~h}$, the cell cytotoxicity was analyzed by MTT assay, and the $\mathrm{IC}_{50}$ was calculated using CalcuSyn (version 2.1). Values shown are the mean \pm standard deviation of quadruplicate determinations. $\mathrm{IC}_{50}$, half maximal inhibitory concentration; Eth, ethoxysanguinarine.
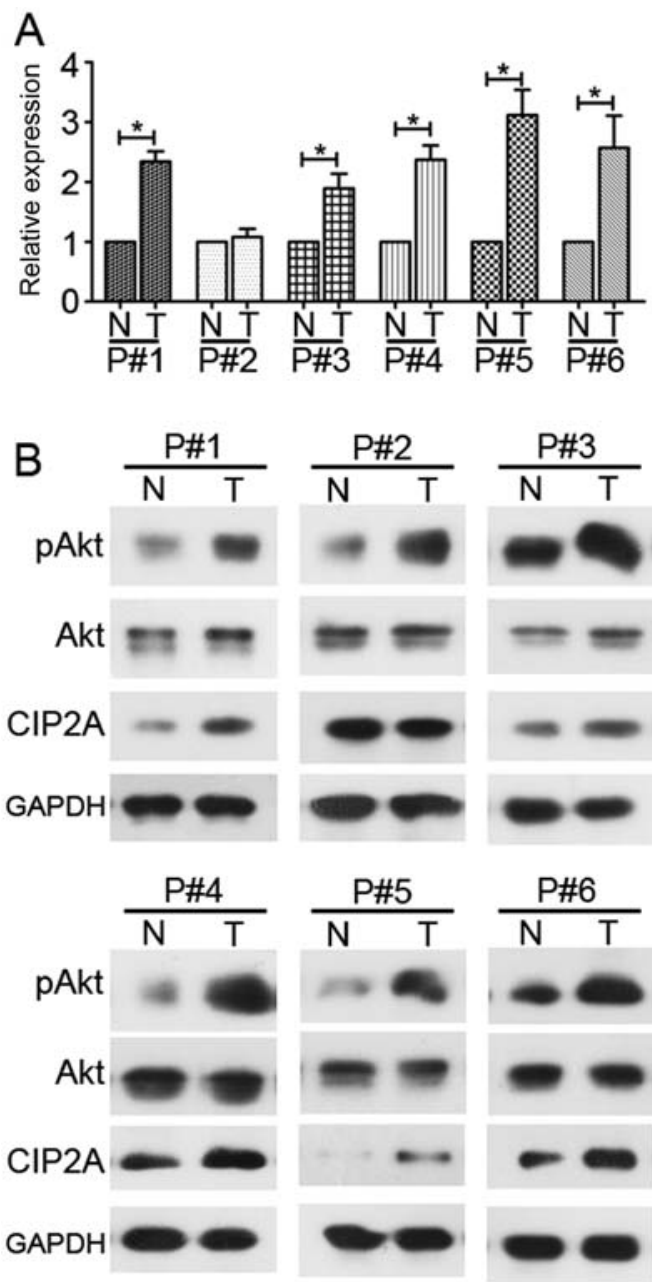

Figure 1. CIP2A expression and Akt phosphorylation in human CRC tissues. (A) The mRNA expression of CIP $2 A$ in 6 human CRC and adjacent normal colonic tissues $\left({ }^{*} \mathrm{P}<0.05\right.$ vs. $\left.\mathrm{N}\right)$. (B) The expression of CIP2A and Akt phosphorylation in 6 human $\mathrm{CRC}$ and adjacent normal colonic tissues as tested by western blot assay. GAPDH was used as the internal control. T, tumor tissues; $\mathrm{N}$, adjacent normal colonic tissues; CRC, colorectal cancer; pAkt, phosphorylated protein kinase $\mathrm{B}$; CIP2A, cancerous inhibitor of protein phosphatase $2 \mathrm{~A}$.

CIP2A-knockdown enhances the inhibition of viability and apoptosis induction effects of Eth. Next, the effect of CIP2A depletion on the inhibition of viability and the induction of apoptosis by Eth were determined. Two siRNAs targeting CIP2A were synthesized and applied to SW620 
A<smiles></smiles>

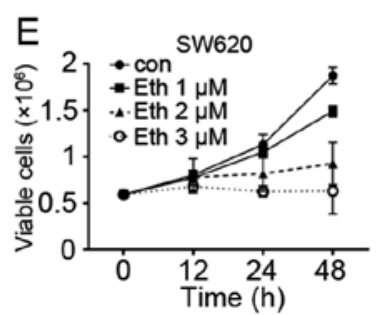

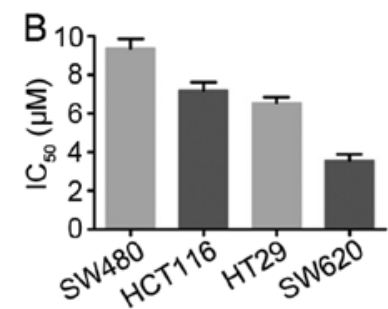

$\mathrm{F}$

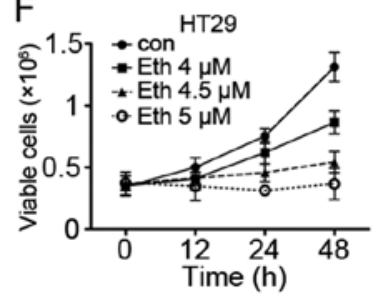

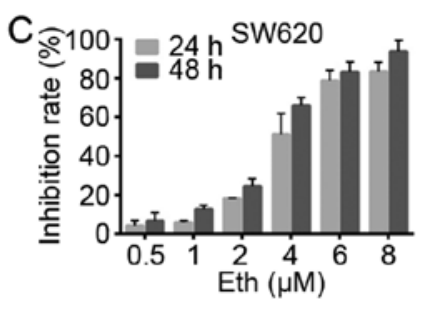
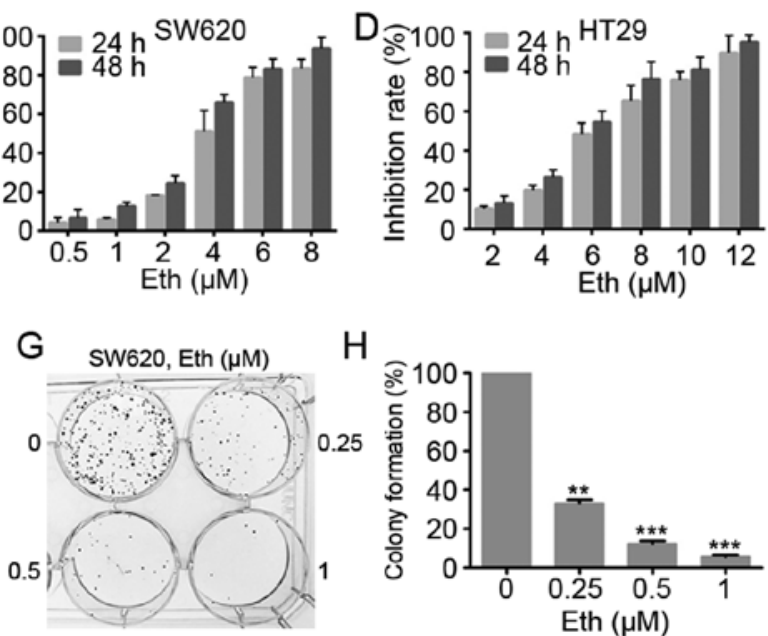

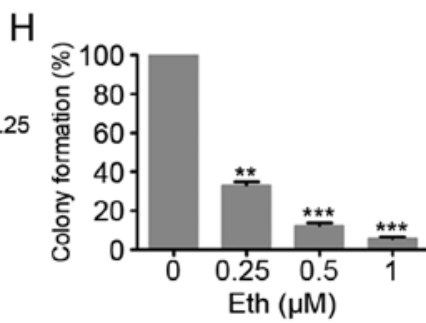

Figure 2. Effect of Eth on SW620 and HT29 colorectal cancer cells. (A) Chemical structure of Eth. (B) The $\mathrm{IC}_{50}$ of Eth for the indicated cell lines. (C and D) The inhibitory effects of Eth on SW620 and HT29 cells were assessed by MTT assay. (E and F) The inhibitory effects of Eth on the cell viability of SW620 and HT29 cells were assayed by trypan blue exclusion assay. ( $\mathrm{G}$ and H) The colony formation assays of SW620 cells treated with Eth at the indicated concentrations. Eth, ethoxysanguinarine; $\mathrm{IC}_{50}$, half maximal inhibitory concentration.
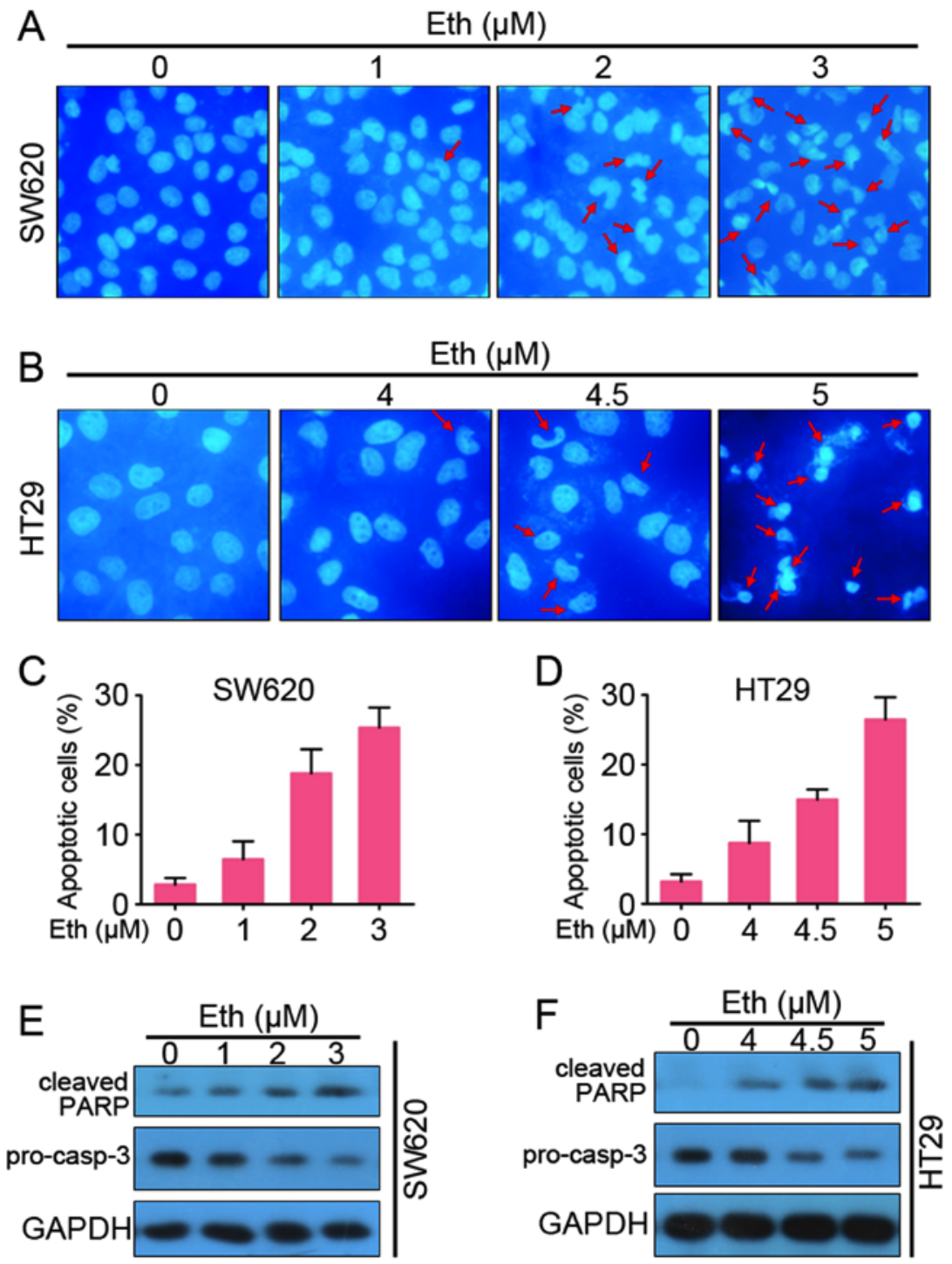

Figure 3. Eth induces apoptosis in colorectal cancer cells. (A and B) SW620 and HT29 cells were incubated with various concentrations of Eth for 24 h. The cells were examined by DAPI staining. (C and D) SW620 and HT29 cells were treated with increasing concentrations of Eth for 24 h, and cell apoptosis was analyzed by Annexin V/propidium iodide staining and flow cytometry. (E and F) SW620 and HT29 cells were treated with increasing concentrations of Eth for $24 \mathrm{~h}$. Western blot analysis was performed using the indicated antibodies. Eth, ethoxysanguinarine; PARP, poly ADP ribose polymerase; casp-3, caspase-3. 

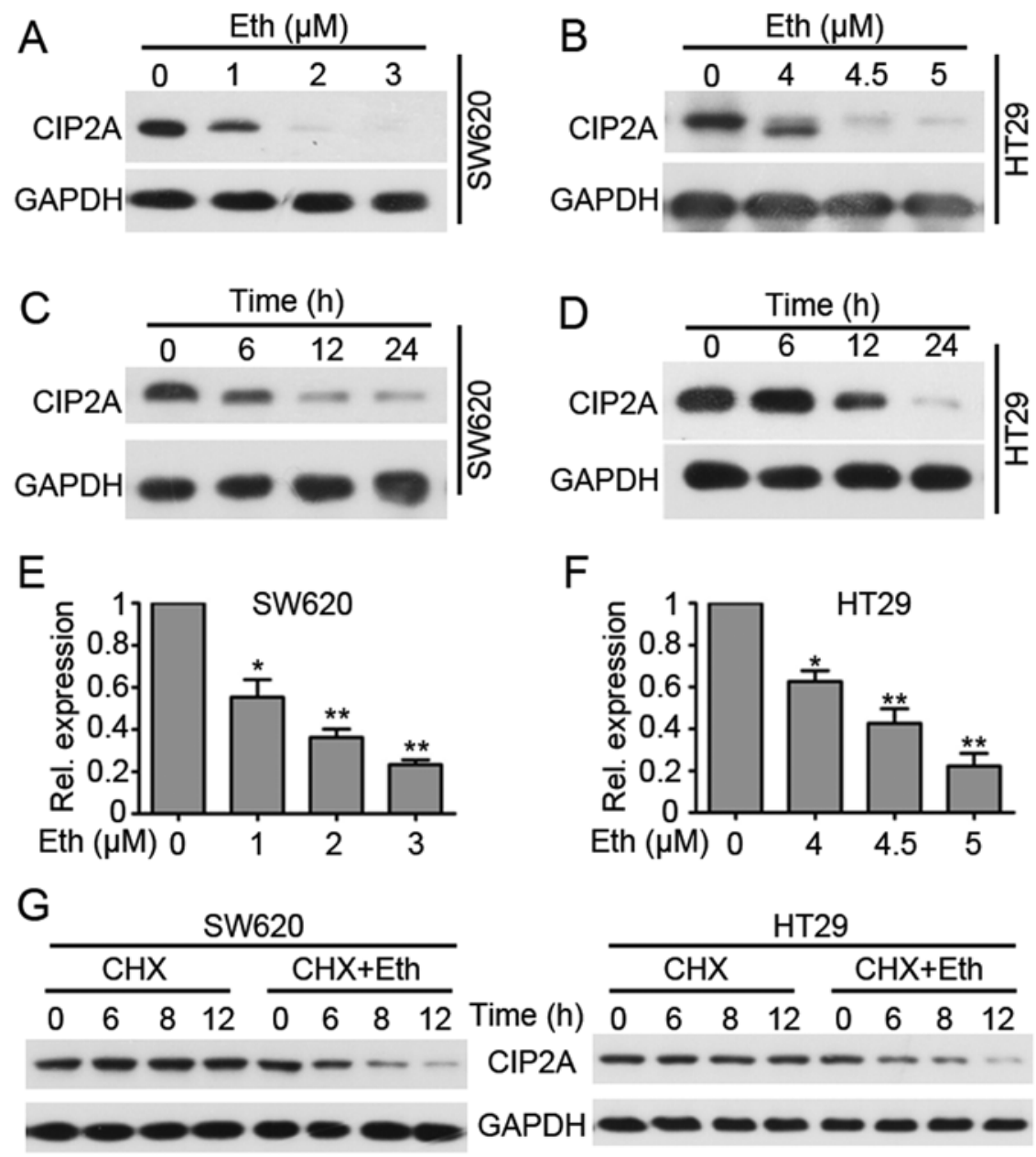

Figure 4. Eth downregulates CIP2A in colorectal cancer cells. (A and B) SW620 and HT29 cells were incubated with Eth at the indicated concentrations for $24 \mathrm{~h}$, followed by western blot analysis using the indicated antibodies. GAPDH was used as a loading control. (C and D) SW620 (or HT29) cells were treated with $2 \mu \mathrm{M}$ (or $4.5 \mu \mathrm{M}$ ) Eth for the indicated times, and cell lysates were subjected to western blot assay. (E and F) The mRNA level of CIP2A in cells treated with Eth for $24 \mathrm{~h}$ was analyzed by quantitative polymerase chain reaction. ${ }^{*} \mathrm{P}<0.05$ and ${ }^{* * *} \mathrm{P}<0.01$ vs. $0 \mu \mathrm{M}$. (G) SW620 (or HT29) cells were treated with $50 \mu \mathrm{g} / \mathrm{ml} \mathrm{CHX}$ in the absence or presence of $2 \mu \mathrm{M}$ (or $4.5 \mu \mathrm{M})$ Eth for the indicated times, and cell lysates were harvested for western blot assay. Eth, ethoxysanguinarine; CIP2A, cancerous inhibitor of protein phosphatase 2A; CHX, cycloheximide.

and HT29 cells. siRNA1 and siRNA2 knockdown considerably decreased CIP2A protein expression in each CRC cell line (Fig. 5A and B). This finding validated the specificity and effectiveness of the CIP2A siRNAs. To examine the effect of CIP2A in Eth-induced inhibition of viability, SW620 and HT29 cells were transfected with CIP2A siRNA and then subjected to Eth treatment. Cell viability and flow cytometric assays were used to detect variations in cell viability and apoptosis. Notably, CIP2A depletion promoted the Eth-induced inhibition of viability (Fig. 5C and D) and enhanced Eth-induced apoptotic effects (Fig. 5E and F). Detection of PARP cleavage suggested that Eth caused a marked rise in PARP cleavage in SW620/siCIP2A and HT29/siCIP2A cells (Fig. 5G).

CIP2A overexpression antagonizes the inhibition of viability and the apoptosis induced by Eth. To certify the functional role of CIP2A in Eth-induced viability inhibition and apoptosis, SW620 and HT29 cells were also transfected with CIP2A plasmid (SW620/CIP2A or HT29/CIP2A), and CIP2A expression was confirmed using western blot analysis (Fig. 6A and B). Cell viability and flow cytometric assays were used to investigate variations in cell viability and apoptosis. Notably, CIP2A overexpression abolished Eth-induced viability inhibition (Fig. 6C and D) and inhibited Eth-induced apoptosis effects (Fig. 6E-G). These data certify the role of CIP2A in Eth-induced viability inhibition and apoptosis.

Eth downregulates the CIP2A/PP2A/Akt pathway. Overexpression of CIP2A causes the activation of PP2A downstream molecule Akt (24). In the present study, the activity of PP2A was next tested, and it was found that PP2A activity was increased in the Eth-treated SW620 and HT29 cells compared with that in the control group (Fig. 7A). Furthermore, the expression of PP2A downstream molecule Akt was examined and it was found that Eth downregulated phospho-Akt in the SW620 and HT29 cells (Fig. 7B). The total Akt level did not markedly change in the Eth-treated SW620 and HT29 cells compared with that in the control group. These results suggest that Eth decreases CIP2A/PP2A/Akt pathway expression in CRC cells.

Eth exhibits antitumor effects on CRC xenograft tumors in vivo. To access the antitumor effect on CRC in vivo, $5 \times 10^{6}$ SW620 cells in $100 \mu \mathrm{l}$ serum-free L-15 medium were 

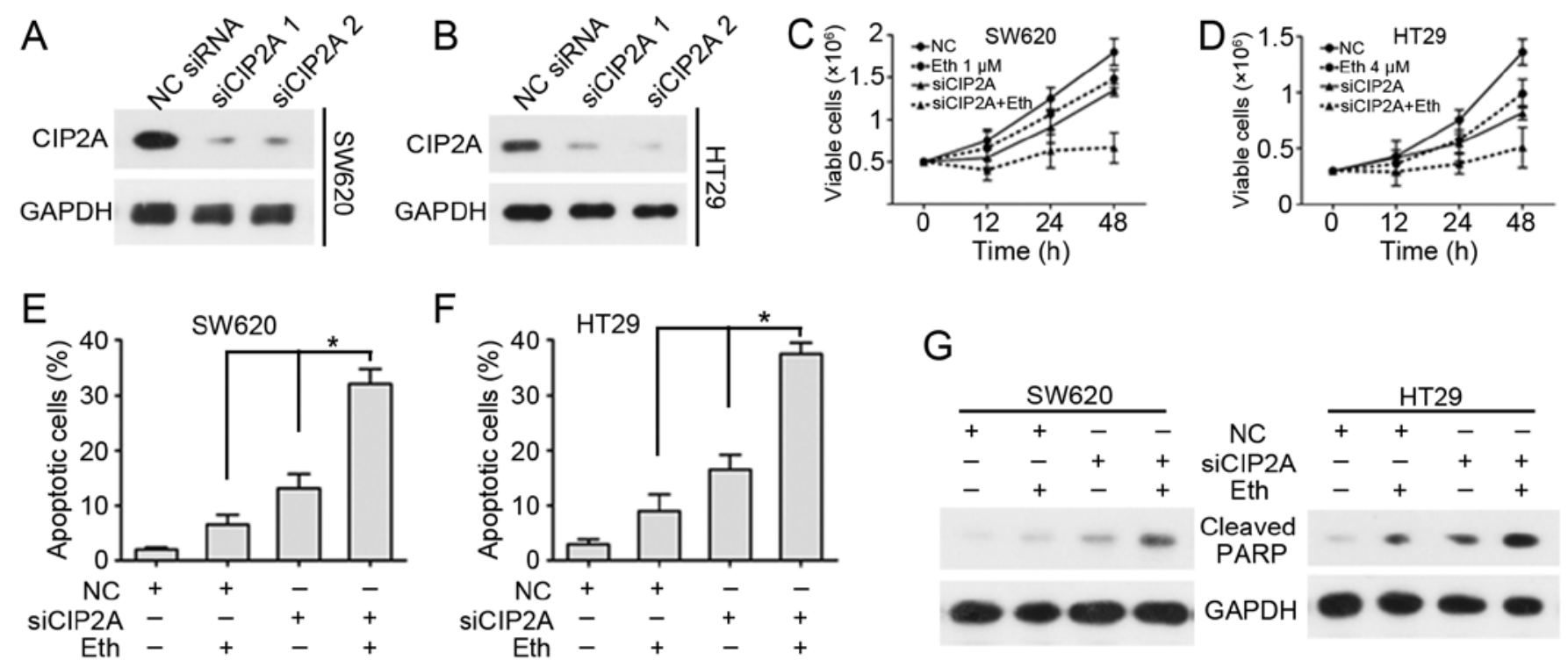

Figure 5. CIP2A-knockdown enhances Eth-induced viability inhibition and apoptosis. (A and B) SW620 and HT29 cells were transfected with $100 \mathrm{nM}$ CIP2A-specifc siRNA or NC siRNA for $48 \mathrm{~h}$. Western blotting of the total cell lysates was performed using specific antibodies as indicated. (C and D) Following transfection with CIP2A-specific siRNA or NC siRNA, SW620 (or HT29) cells were treated with $1 \mu \mathrm{M}$ (or $4 \mu \mathrm{M})$ Eth for the indicated times. (E and F) SW620 (or HT29) cells were transfected with CIP2A-specific siRNA or NC siRNA, followed by treatment with $1 \mu \mathrm{M}$ (or $4 \mu \mathrm{M}$ ) Eth for $24 \mathrm{~h}$. Cell apoptosis was analyzed by Annexin V/propidium iodide staining and flow cytometry. "P<0.05. (G) SW620 (or HT29) cells were transfected with CIP2A-specific siRNA or NC siRNA, followed by treatment with $1 \mu \mathrm{M}$ (or $4 \mu \mathrm{M}$ ) Eth for $24 \mathrm{~h}$. Cells were collected for western blot analyses. Eth, ethoxysanguinarine; CIP2A, cancerous inhibitor of protein phosphatase $2 \mathrm{~A}$; si, small interfering; $\mathrm{NC}$, negative control.
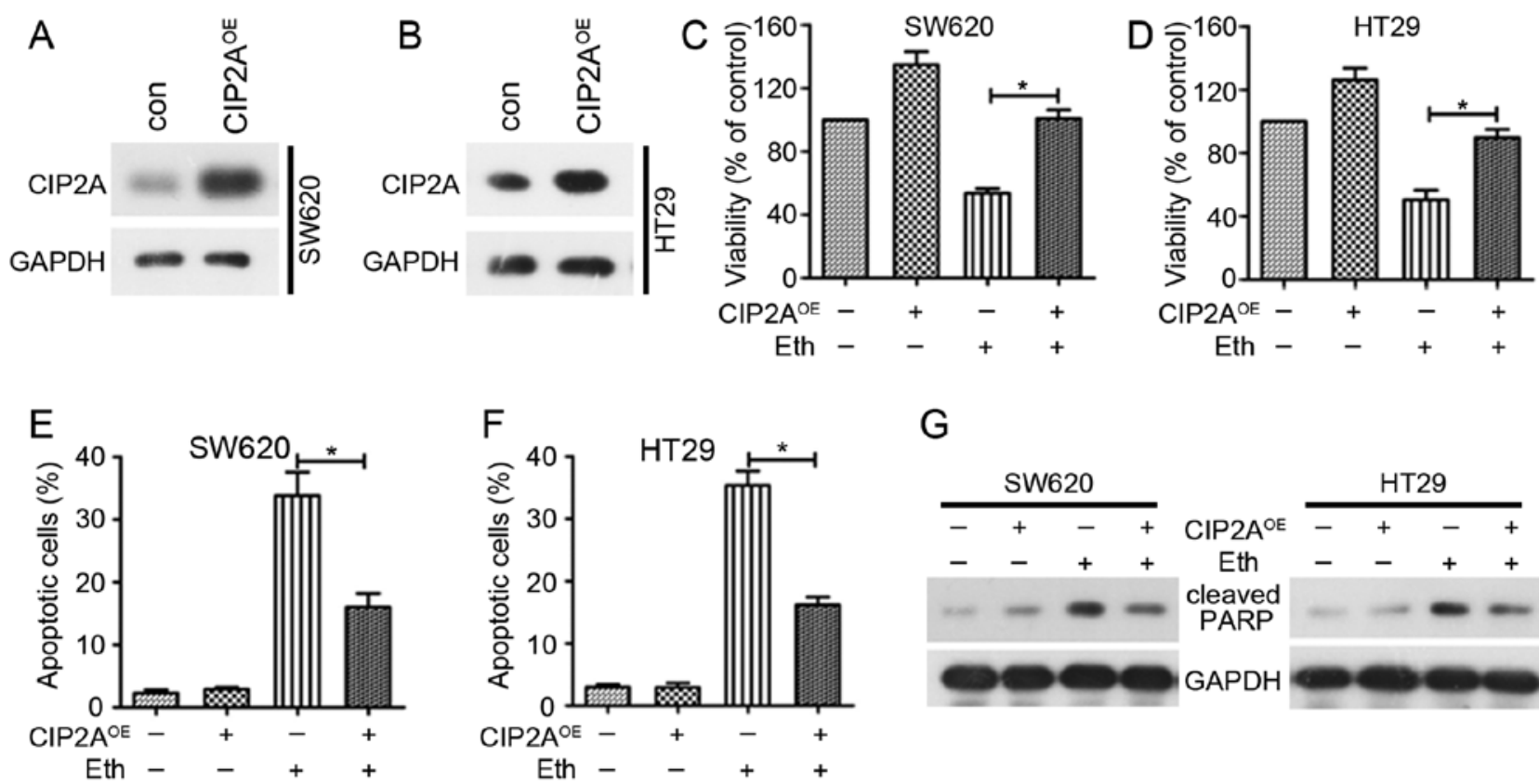

G

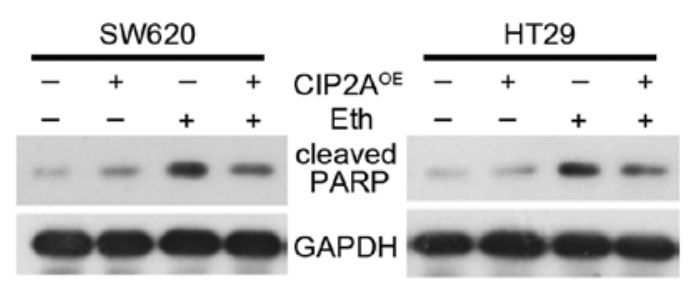

Figure 6. Overexpression of CIP2A reduces Eth-induced viability inhibition and apoptosis. (A and B) SW620 and HT29 cells were transfected with the pOTENT-1-CIP2A expression plasmid (CIP2 $\mathrm{A}^{\mathrm{OE}}$ ), and then western blotting was used to detect CIP2A expression at $48 \mathrm{~h}$ post-transfection. (C and D) SW620 (or HT29) cells were transfected with CIP2 $\mathrm{A}^{\mathrm{OE}}$, and after $6 \mathrm{~h}$ of transfection, the cells were treated with $4 \mu \mathrm{M}$ (or $6 \mu \mathrm{M}$ ) Eth for $24 \mathrm{~h}$. MTT assay were used to detect the viability of the cells. (E and F) SW620 (or HT29) cells were transfected with CIP2A $\mathrm{A}^{\mathrm{OE}}$, and after $6 \mathrm{~h}$ of transfection, the cells were treated with $4 \mu \mathrm{M}$ (or $6 \mu \mathrm{M}$ ) Eth for $24 \mathrm{~h}$. Cells were stained with Annexin V/propidium iodide for apoptosis analyses. "P<0.05. (G) SW620 (or HT29) cells were transfected with $\mathrm{CIP}_{2} \mathrm{~A}^{\mathrm{OE}}$, and after $6 \mathrm{~h}$ of transfection, the cells were treated with $3 \mu \mathrm{M}$ (or $5 \mu \mathrm{M}$ ) Eth for $24 \mathrm{~h}$. Cells were then lysed for western blotting using the indicated antibodies. Eth, ethoxysanguinarine; CIP2A, cancerous inhibitor of protein phosphatase 2A; CIP2A ${ }^{\mathrm{OE}}$, CIP2A overexpression plasmid.

subcutaneously inoculated into the right flank of nude mice to generate xenografted murine models. When the tumors grew to a measurable size, each group (10 mice for each random allocation) was administrated with vehicle control (0.5\% DMSO/10\% Cremophor/5\% ethanol in normal saline) or
Eth $(0.5 \mathrm{mg} / \mathrm{kg}) 5$ times per week for 4 weeks. Tumor-bearing mice were humanely sacrificed when their tumors reached $1.5 \mathrm{~cm}$ in diameter or when paralysis or a major compromise in their quality of life occurred. As expected, it was found that Eth efficiently repressed tumor growth compared with the vehicle 

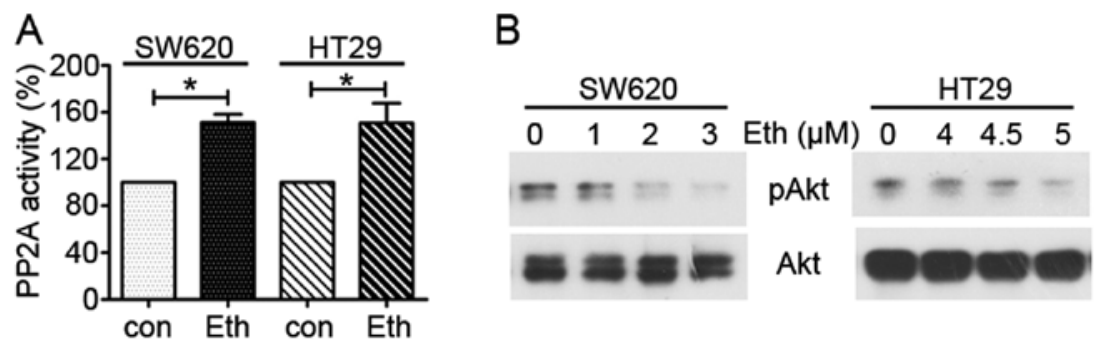

Figure 7. Effects of Eth on CIP2A downstream molecules. (A) SW620 (or HT29) cells were treated with $3 \mu \mathrm{M}$ (or $5 \mu \mathrm{M}$ ) Eth for $24 \mathrm{~h}$ and then cell lysates were prepared for detecting PP2A activity as aforementioned. ${ }^{*} \mathrm{P}<0.05$. (B) SW620 and HT29 cells were treated with Eth at the indicated concentrations for $24 \mathrm{~h}$, followed by western blot analysis using the indicated antibodies. Eth, ethoxysanguinarine; CIP2A, cancerous inhibitor of protein phosphatase 2A; Con, control; pAkt, phosphorylated protein kinase B; PP2A, protein phosphatase 2A.

A

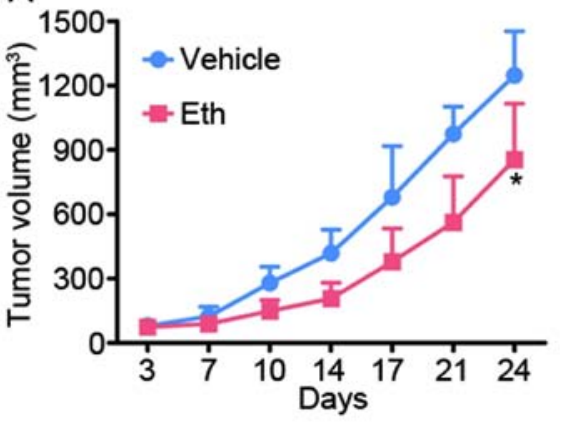

C

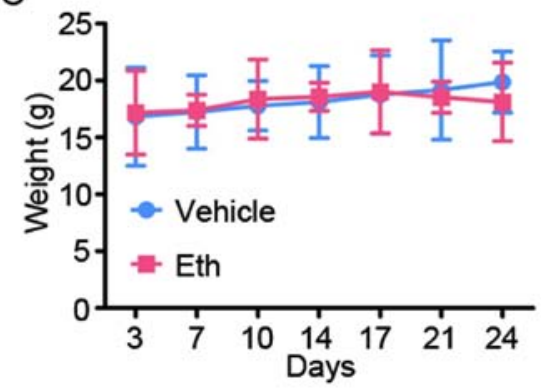

B

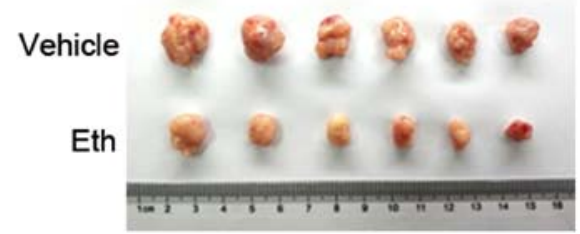

D

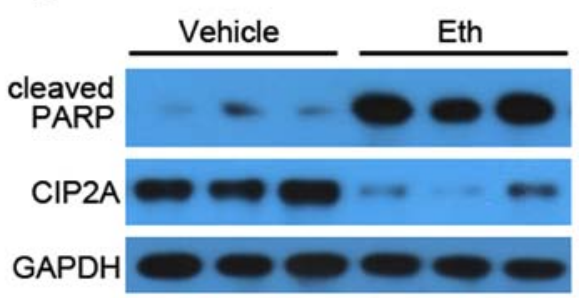

Figure 8. In vivo therapeutic efficacy of Eth on a human colorectal cancer murine model. (A) Murine models were treated with vehicle or $0.5 \mathrm{mg} / \mathrm{kg}$ Eth, and the tumor volumes were calculated twice a week. ${ }^{*} \mathrm{P}<0.05$ vs. vehicle. (B) Images of xenograft tumors obtained from mice with different treatments after 4 weeks. (C) Eth treatment did not affect the murine model body weight. (D) The expression of the indicated proteins in the xenograft tumors was analyzed by western blotting. Eth, ethoxysanguinarine; CIP2A, cancerous inhibitor of protein phosphatase 2A; PARP, poly ADP ribose polymerase.

control $(\mathrm{P}<0.05)$ (Fig. 8A and B). Additionally, Eth treatment did not reduce the body weight of the mice, which suggested that Eth had no apparent side effect (Fig. 8C). All the mice were euthanized, the tumors were isolated and imaged, and the tumor cell samples were harvested to extract protein for determination of whether Eth inhibited CIP2A and induced apoptosis, as determined via western blotting. As shown in Fig. 8D, the level of CIP2A in the Eth treatment group was decreased compared with that in the vehicle control. Meanwhile, consistent with the aforementioned results, the level of cleaved-PARP in the Eth treatment group was increased compared with that in the vehicle control. These results suggested that Eth has immense potential for CRC therapy.

\section{Discussion}

The present study found that Eth treatment caused a decrease in CIP2A expression in SW620 and HT29 CRC cells, which was associated with the inhibition of cell viability and the induction of apoptosis. Furthermore, it was demonstrated that CIP2A-knockdown sensitized Eth-induced cellular effects, and conversely, that CIP2A overexpression antagonized Eth-induced viability inhibition and apoptosis. These data suggested that Eth specifically affects CIP2A in CRC cells and that the decrease in CIP2A expression may serve a key role in its antitumor effects. This study identifies Eth as a novel CIP2A inhibitor and provides data to improve our understanding of the inhibition of viability and induction of apoptosis caused by Eth.

Previous research has indicated that CIP2A is a potential diagnostic and prognostic marker of several cancer types, including CRC $(25,26)$. In the present study, the expression of CIP2A was examined in 6 human CRC cases by western blotting and qPCR (Fig. 1). The results showed that the mRNA and protein expression of CIP2A in CRC tissues was increased in comparison with that in adjacent normal tissue, indicating that CIP2A serves a role in the tumorigenesis of CRC.

Natural products continue to be targeted as a novel dependable source for the treatments of numerous fatal diseases, 
such as cancer, due to their preferential properties, including reduced toxicity, the ability to elicit fewer adverse side effects compared with synthetic compounds, their high bioavailability and their reliability (10). M. cordata is a perennial herb that belongs to the Papaveraceae family, which is distributed in hills or low mountains, at an elevation of 150-830 meters above sea level in China (27). The Shennongjia National Nature Reserve has abundant distribution of this species. M. cordata has been approved by the European Food Safety Authority (EFSA) as a safe plant for the manufacture of feed additives, suggesting its low toxicity. M. cordata is a perfect substitute for bloodroot as a commercial source for the industrial production of sanguinarine (27). In the present study, it was demonstrated that Eth, a derivative of sanguinarine, significantly inhibited CRC cell viability and colony formation at low micromolar concentrations, suggesting its potential for use in CRC control (Fig. 2).

One of the most efficient strategies in cancer therapy is the induction of cell apoptosis. In the present study, Eth was found to induce nuclear shrinkage and chromatin condensation (Fig. 3A and B), indicating that Eth induces apoptosis in CRC cells. Furthermore, using flow cytometry, 3 and $5 \mu \mathrm{M}$ Eth was found to cause apoptosis at a ratio of 25.39 and $26.49 \%$ in SW620 and HT29 cells, respectively (Fig. 3C and D). These results further indicated that Eth induces apoptosis in CRC cells. The extrinsic and intrinsic apoptotic pathways finally result in the activation of effector caspases (casp)-3,-2 and -7. The latter is associated with PARP degradation; a hallmark of apoptosis due it being a substrate of activated effector caspases $(28,29)$. The potential association between Eth-induced apoptosis and caspase activation was also examined (Fig. 3E and F). Thus, Eth may induce apoptosis by activating the apoptosis pathway, which leads to the activation of effector casp-3.

Significant inhibition by Eth of CIP2A expression at the transcriptional and translational levels has been reported in lung cancer cells (12). However, the effects of Eth on CIP2A expression in other cancer types have not been determined and require further investigation. The present study found that Eth was also able to induce a marked dose- and timedependent reduction of CIP2A at the mRNA and protein levels in CRC (Fig. 4). Moreover, it was shown that the depletion of CIP2A enhanced the growth inhibition and apoptotic effects of Eth in CRC (Fig. 5). Additionally, overexpression of CIP2A antagonized the inhibition of viability and the apoptosis induced by Eth (Fig. 6). The activity of CIP2A downstream molecules PP2A and Akt was detected, and it was demonstrated that Eth upregulated PP2A activity and downregulated phospho-Akt, but not total Akt, in SW620 and HT29 (Fig. 7) cells. The dephosphorylation of Akt is frequently mediated by PP2A. In a xenograft murine model of SW620, Eth significantly inhibited tumor growth, but the body weight of the mice remained unaffected (Fig. 8A-C). The results also showed that Eth inhibited CIP2A and induced apoptosis in vivo (Fig. 8D).

In conclusion, the present study demonstrated the mechanism by which Eth induced cancer cell apoptosis in CRC, which was at least in part by inhibiting the CIP2A/PP2A/Akt signaling cascades.

\section{Acknowledgements}

Not applicable.

\section{Funding}

This study was supported by grants from the National Natural Science Foundation of China (no. 81702930), the Open Ended Design Project of the Hubei Province Key Laboratory of Conservation Biology for Shennongjia Golden Monkey (no. 2016SNJ001), the Natural Science Foundation of Hubei Provincial Department of Education (no. B2014048), the Key Discipline Project of Hubei Province; the Foundation of Health and Family planning Commission of Hubei Province (nos. WJ2017F065 and WJ2017F067) and the Foundation of Hubei University of Medicine (no. FDFR201605).

\section{Availability of data and materials}

Materials described in the manuscript will be freely available to any scientist wishing to use them for non-commercial purposes, without breaching participant confidentiality.

\section{Authors' contributions}

The project was conceived and designed by YG and YL. Patient specimens were harvested/provided by ZL and MX. The experiments were conducted by LJ, YS, XH, PL, BZ, HY, $\mathrm{XZ}$ and SQ. Data were analyzed by YG and LJ. The manuscript was written by YG and YL.

\section{Ethics approval and consent to participate}

All animal studies were conducted according to protocols approved by the Hubei University of Medicine Animal Care and Use Committee (approval no. 2017-56), complying with the rules of Regulations for the Administration of Affairs Concerning Experimental Animals (Approved by the State Council of China). Written informed consent was obtained from all patients.

\section{Consent for publication}

Each patient provided written informed consent.

\section{Competing interests}

The authors declare that they have no competing interests.

\section{References}

1. Torre LA, Bray F, Siegel RL, Ferlay J, Lortet-Tieulent J and Jemal A: Global cancer statistics, 2012. CA Cancer J Clin 65: 87-108, 2015.

2. Chen W, Zheng R, Baade PD, Zhang S, Zeng H, Bray F, Jemal A, $\mathrm{Yu}$ XQ and He J: Cancer statistics in China, 2015. CA Cancer J Clin 66: 115-132, 2016.

3. Jemal A, Center MM, DeSantis C and Ward EM: Global patterns of cancer incidence and mortality rates and trends. Cancer Epidemiol Biomarkers Prev 19: 1893-1907, 2010.

4. Jonas S, Thelen A, Benckert C, Spinelli A, Sammain S, Neumann U, Rudolph B and Neuhaus P: Extended resections of liver metastases from colorectal cancer. World J Surg 31: 511-521, 2007.

5. Pericàs JM, Corredoira J and Miró JM; Hospital Clínic-Lucus Augusti Working Group for endocarditis: Colorectal adenomas. N Engl J Med 375: 387-388, 2016.

6. Junttila MR, Puustinen P, Niemelä M, Ahola R, Arnold H, Böttzauw T, Ala-aho R, Nielsen C, Ivaska J, Taya Y, et al: CIP2A inhibits PP2A in human malignancies. Cell 130: 51-62, 2007. 
7. Li W, Ge Z, Liu C, Liu Z, Björkholm M, Jia J and Xu D: CIP2A is overexpressed in gastric cancer and its depletion leads to impaired clonogenicity, senescence, or differentiation of tumor cells. Clin Cancer Res 14: 3722-3728, 2008.

8. Ren J, Li W, Yan L, Jiao W, Tian S, Li D, Tang Y, Gu G, Liu H and $\mathrm{Xu} \mathrm{Z}$ : Expression of CIP2A in renal cell carcinomas correlates with tumour invasion, metastasis and patients' survival. Br J Cancer 105: 1905-1911, 2011.

9. Liu CY, Shiau CW, Kuo HY, Huang HP, Chen MH, Tzeng CH and Chen KF: Cancerous inhibitor of protein phosphatase $2 \mathrm{~A}$ determines bortezomib-induced apoptosis in leukemia cells. Haematologica 98: 729-738, 2013.

10. Cai F, Zhang L, Xiao X, Duan C, Huang Q, Fan C, Li J, Liu X, Li S and Liu Y: Cucurbitacin B reverses multidrug resistance by targeting CIP2A to reactivate protein phosphatase $2 \mathrm{~A}$ in MCF-7/ adriamycin cells. Oncol Rep 36: 1180-1186, 2016.

11. Perrotti D and Neviani P: Protein phosphatase 2A: A target for anticancer therapy. Lancet Oncol 14: e229-e238, 2013.

12. LiuZ,MaL,WenZS,Cheng YXandZhouGB:Ethoxysanguinarine induces inhibitory effects and downregulates CIP2A in lung cancer cells. ACS Med Chem Lett 5: 113-118, 2013.

13. Kosina P, Gregorova J, Gruz J, Vacek J, Kolar M, Vogel M, Roos W, Naumann K, Simanek V and Ulrichova J: Phytochemical and antimicrobial characterization of Macleaya cordata herb. Fitoterapia 81: 1006-1012, 2010.

14. Ouyang L, Su X, He D, Chen Y, Ma M, Xie Q and Yao S: A study on separation and extraction of four main alkaloids in Macleaya cordata (Willd) $\mathrm{R}$. Br. with strip dispersion hybrid liquid membrane. J Sep Sci 33: 2026-2034, 2010

15. Yao JY, Shen JY, Li XL, Xu Y, Hao GJ, Pan XY, Wang GX and Yin WL: Effect of sanguinarine from the leaves of Macleaya cordata against Ichthyophthirius multifiliis in grass carp (Ctenopharyngodon idella). Parasitol Res 107: 1035-1042, 2010.

16. Konda Y, Urano M, Harigaya Y and Onda M: Studies on the constituents of bocconia-cordata 0.4. Transformation of sanguinarine into bocconine. J Heterocycl Chem 28: 1841-1843, 1991.

17. Livak KJ and Schmittgen TD: Analysis of relative gene expression data using real-time quantitative PCR and the 2(-Delta Delta C(T)) method. Methods 25: 402-408, 2001.

18. Cao W, Liu Y, Zhang R, Zhang B, Wang T, Zhu X, Mei L, Chen H, Zhang H, Ming P, et al: Homoharringtonine induces apoptosis and inhibits STAT3 via IL-6/JAK1/STAT3 signal pathway in Gefitinib-resistant lung cancer cells. Sci Rep 5: 8477, 2015.
19. Liu X, Duan C, Ji J, Zhang T, Yuan X, Zhang Y, Ma W, Yang J, Yang L, Jiang Z, et al: Cucurbitacin B induces autophagy and apoptosis by suppressing CIP2A/PP2A/mTORC1 signaling axis in human cisplatin resistant gastric cancer cells. Oncol Rep 38 : 271-278, 2017.

20. Feng T, Cao W, Shen W, Zhang L, Gu X, Guo Y, Tsai HI, Liu X, Li J, Zhang J, et al: Arctigenin inhibits STAT3 and exhibits anticancer potential in human triple-negative breast cancer therapy. Oncotarget 8: 329-344, 2017.

21. Chou CC, Yang JS, Lu HF, Ip SW, Lo C, Wu CC, Lin JP, Tang NY, Chung JG, Chou MJ, et al: Quercetin-mediated cell cycle arrest and apoptosis involving activation of a caspase cascade through the mitochondrial pathway in human breast cancer MCF-7 cells. Arch Pharm Res 33: 1181-1191, 2010.

22. Liu Y, Dong Y, Zhang B and Cheng YX: Small compound 6-O-angeloylplenolin induces caspase-dependent apoptosis in human multiple myeloma cells. Oncol Lett 6: 556-558, 2013.

23. Liu H, Gu Y, Wang H, Yin J, Zheng G, Zhang Z, Lu M, Wang C and He Z: Overexpression of PP2A inhibitor SET oncoprotein is associated with tumor progression and poor prognosis in human non-small cell lung cancer. Oncotarget 6: 14913-14925, 2015.

24. Rincón R, Cristóbal I, Zazo S, Arpí O, Menéndez S, Manso R, Lluch A, Eroles P, Rovira A, Albanell J, et al: PP2A inhibition determines poor outcome and doxorubicin resistance in early breast cancer and its activation shows promising therapeutic effects. Oncotarget 6: 4299-4314, 2015.

25. Cristóbal I, Zazo S, Torrejón B, Pedregal M, Madoz-Gúrpide J, Lluch A, Eroles P, Rovira A, Albanell J, García-Foncillas J, et al: CIP2A confirms its prognostic value in triple-negative breast cancer. Oncogene 36: 3357-3358, 2017.

26. Balliu M,Cellai C,Lulli M,Laurenzana A,Torre E, Vannucchi AM and Paoletti F: HDAC1 controls CIP2A transcription in human colorectal cancer cells. Oncotarget 7: 25862-25871, 2016.

27. Huang Q, Qin S, Yuan X, Zhang L, Ji J, Liu X, Ma W, Zhang Y, Liu P, Sun Z, et al: Arctigenin inhibits triple-negative breast cancers by targeting CIP $2 \mathrm{~A}$ to reactivate protein phosphatase $2 \mathrm{~A}$. Oncol Rep 38: 598-606, 2017.

28. Nicholson DW: Caspase structure, proteolytic substrates, and function during apoptotic cell death. Cell Death Differ 6: 1028-1042, 1999.

29. Johnstone RW, Ruefli AA and Lowe SW: Apoptosis: A link between cancer genetics and chemotherapy. Cell 108: 153-164, 2002. 